\title{
Narrow Frequency Range Problem of Sound Reflector Arrays
}

\author{
A. SzeląG, J. Rubacha And T. KAMisiński*
}

AGH - University of Science and Technology, Faculty of Mechanical Engineering and Robotics

Department of Mechanics and Vibroacoustics, al. A. Mickiewicza 30, 30-059 Krakow, Poland

Overhead stage canopies composed of many panels suspended under ceiling provide proper acoustic field in concert halls and auditoria. The purpose of using these structures is adequate direction and partial dispersion of sound reflected from them. Frequency range of sound reflected from flat panels closely depends on their shape, size and configuration. It is often too narrow and therefore insufficient for the proper sound of the interior. Mutual dependence of the lower and upper frequency limit of sound transmission requires the search for other ways to improve these structures. The paper proposes some solutions concerning spatial structures which have not been yet defined. An attempt was made to determine the useful frequency band of sound transmitted through such reflective structures.

DOI: $10.12693 /$ APhysPolA.123.1059

PACS: 43.55.-n, 43.20.El

\section{Introduction}

Overhead stage canopies (Fig. 1) composed of many panels suspended from the ceiling provide proper acoustic field in concert halls and auditoria. The purpose of using these structures is adequate direction and partial dispersion of sound reflected from them. The frequency response in the range from $250 \mathrm{~Hz}$ to $4 \mathrm{kHz}$ is required and the spectrum in this range should be flat $( \pm 3 \mathrm{~dB})$ and vary little from place to place.

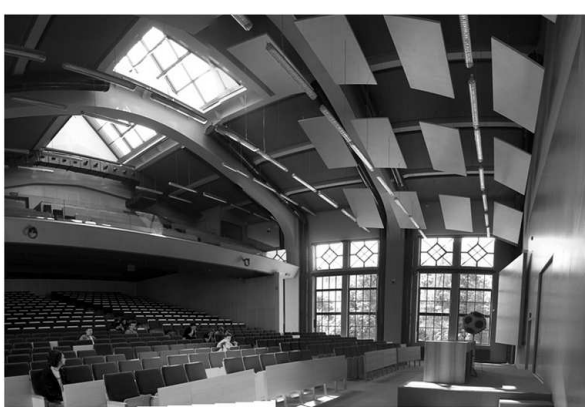

Fig. 1. Reflection panels in conference hall, AGH University of Science and Technology, Cracow.

The frequency response of a reflection array may be described as this of a high pass filter and represented by a relationship between the relative sound reflection level and the frequency (Fig. 2) [1, 2].

There are two independent low frequency limits. One is caused by attenuation of wavelengths large compared to dimensions of the array's element [3]. The other is due to attenuation from diffraction [4]. Nevertheless, Skålevik $[1,2]$ proposed a simplified formula of low frequency limit deducted from the scattering theory:

*corresponding author; e-mail: kamisins@agh.edu.pl

$$
f_{\text {low }}=64 \varepsilon,
$$

where $\varepsilon$ (panel edge density):

$$
\varepsilon=\frac{l_{\text {panel }}}{S_{\text {panel }}}
$$

where $l_{\text {panel }}$ is the panel edge lenght and $S_{\text {panel }}$ is the single panel surface area.

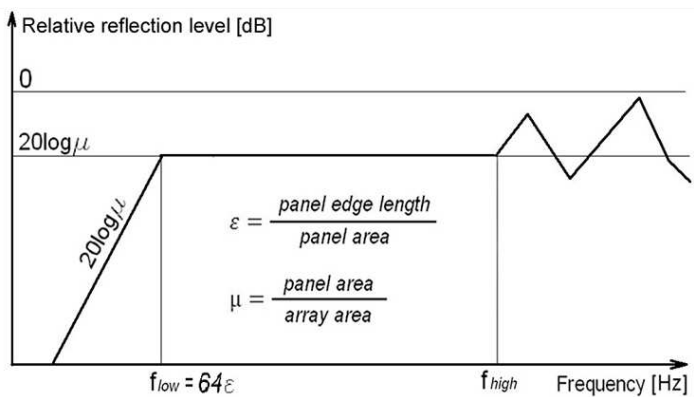

Fig. 2. Frequency response of reflection array dependent on panel density $\mu$ and panel edge density $\varepsilon$.

It is also necessary to determine the high frequency limit, since above this boundary the reflection level depends on whether the geometric reflection point is on element or in between. The appropriate formula for high frequency limit was given by Rindel [4]:

$$
f_{\text {high }}=\frac{c a *}{2 S_{\text {panel }} \cos \Theta},
$$

where $c$ is the speed of sound, $\Theta$ is the angle of incidence and $a *$ is the characteristic distance determined by the formula:

$$
a *=\frac{2 a_{1} a_{2}}{a_{1}+a_{2}},
$$

where $a_{1}$ is the distance from reflector to sound source and $a_{2}$ is the distance from reflector to receiver.

For practical reasons, the most commonly used are flat panel reflector arrays. Unfortunately, the frequency range of sound reflected from such structures is often too narrow and therefore insufficient for the proper sound 
quality of the interior. For example, to provide response in the $250 \mathrm{~Hz}$ octave, square reflector panels should be at least $1 \mathrm{~m} \times 1 \mathrm{~m}$ in size. Assuming the position of structure $8 \mathrm{~m}$ over the stage, high frequency limit calculated from Eq. (3) is $1360 \mathrm{~Hz}$, which is lower than recommended.

Mutual dependence of the lower and upper frequency limit of sound transmission requires the search for other ways to improve these structures. The paper proposes some solutions concerning spatial structures which have not yet been defined. An attempt was made to determine the useful frequency band of sound transmitted through such reflective structures.

\section{Measurements}

To study reflection structure efficiency and limit frequencies of selected reflection structures in practice, a measurement setup was designed and situated in anechoic chamber (Fig. 3). The examined model elements were made of fibreboard and reflected all analysed frequencies sufficiently. For normal incidence of sound waves, impulse responses were measured and further calculations may be expressed by following formula [5]:

$$
L_{\mathrm{x}}=20 \log \left(\frac{\mathcal{F}\left(h(t)_{\text {array }}-h(t)_{\text {empty }}\right)}{\mathcal{F}\left(h(t)_{\text {ref }}-h(t)_{\text {empty }}\right)}\right),
$$

where $\mathcal{F}$ is the Fourier transform, $h(t)_{\text {array }}$ is the impulse response of reflection array, $h(t)_{\text {ref }}$ is the impulse response of reference ( $100 \%$ array density, $\mu=1$ ) and $h(t)_{\mathrm{empty}}$ is the impulse response of measurement setup without tested structures.

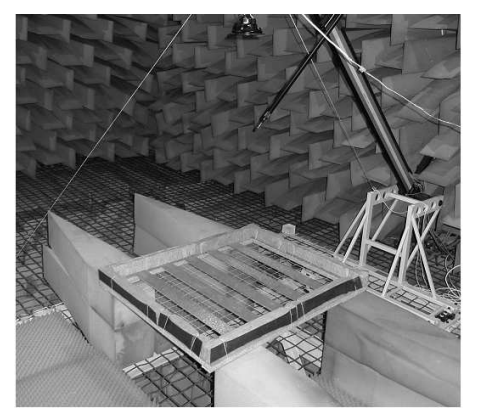

Fig. 3. Researched structure on the measurement setup in anechoic chamber.

The obtained frequency responses were compared in terms of cut-off frequencies and the characteristic's smoothness in the bandwidth. In order to clearly describe the smoothness of frequency response, the peakto-peak value was used defined by following formula:

$$
L_{\mathrm{pp}}=L_{\max }-L_{\min },
$$

where $L_{\max }$ is the maximum relative sound reflection level in the bandwidth and $L_{\min }$ is the minimum relative sound reflection level in the bandwidth.

The limit value of above index was defined as $6 \mathrm{~dB}$. Below this value, the concerned frequency response is consider to be even.

\section{Experiments and results}

\subsection{The effect of element shape}

The narrow frequency range problem of flat reflector arrays might be solved by choosing the appropriate shape of panels. Analyzing formulas (1) and (2), it can be noted that low frequency limit depends only on single panel's shape. For square, circular, and triangular reflective elements, respectively, panel edge densities may be described as follows:

$$
\varepsilon_{\text {squares }}=\frac{4}{a}, \varepsilon_{\text {circles }}=\frac{4}{d}, \varepsilon_{\text {triangles }}=\frac{4 \sqrt{3}}{a}=\frac{6}{h},
$$

where $a$ is the length of squares' and triangles' sides, $d$ is the circles' diameter, and $h$ is the triangles' height.

Therefore, among elements of the same dimensions, square panels are preferred due to the lowest cut-off frequency and easiness of realization. However, it is necessary to verify the smoothness of their pass band. Ando [6] suggested that peaks and dips in frequency response also depend on panel geometry. Applying the diffraction integral of Rubinowicz, he calculated the transfer function for reflection produced by canopy panels of various shapes. Afterwards, he compared graphs obtained for triangular, rectangular and decagon panels, suggesting that the first ones were the most favorable.

Since the theoretical model has a number of restrictions and simplifications, the present authors have proposed experimental study on similar model elements (Fig. 4).

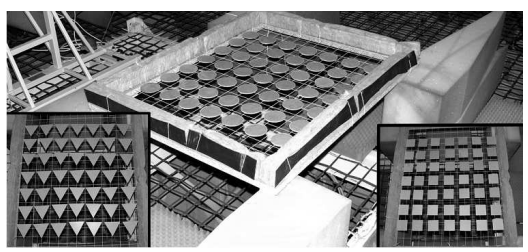

Fig. 4. Arrays composed of circular, triangular and square elements on the measurement setup.

The results of the research on the discussed elements are shown in Fig. 5. Dimensions of the considered elements are similar thus low frequency limits of square and circular panels should be also similar whereas the cut-off frequency for triangular elements is predicted to be higher. Nonetheless, it is not exactly noticeable in Fig. 5. While responses of square and circular panels are in accordance with assumptions, the cut-off frequency for triangular elements is too low. It may be supposed that the shape of reflection elements influences the range of frequency response, therefore the proposed formula (1) is not appropriate for all sorts of panels. It might be also noticed that all arrays mentioned above have similar smoothness of the frequency response with an evident peak around the cut-off frequency. Thus, the experiment does not confirm the Ando's statement. In practice, much smoother frequency response might be obtained by uneven arrangement of the panels used (Fig. 6). 


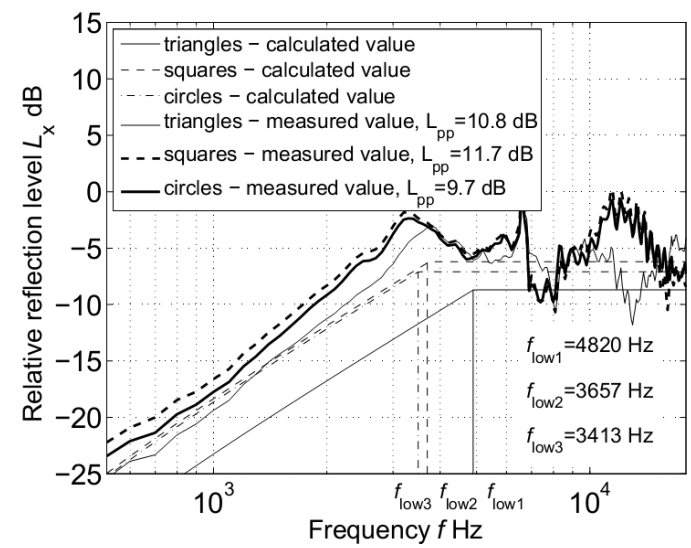

Fig. 5. Frequency responses of reflection arrays composed of triangle, circular or square elements.

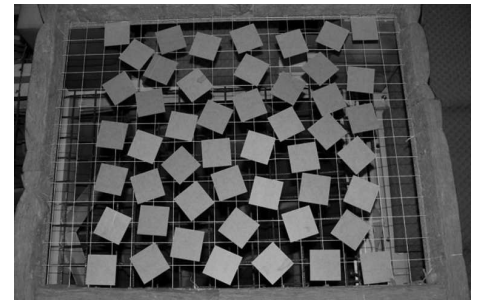

Fig. 6. An array composed of irregularly arranged square elements on the measurement setup.

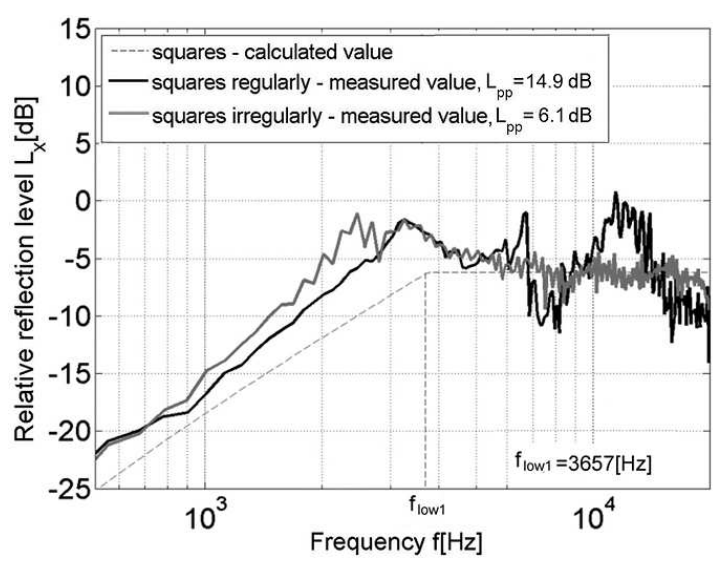

Fig. 7. Frequency responses of reflection arrays composed of square elements in regular and irregular arrangement.

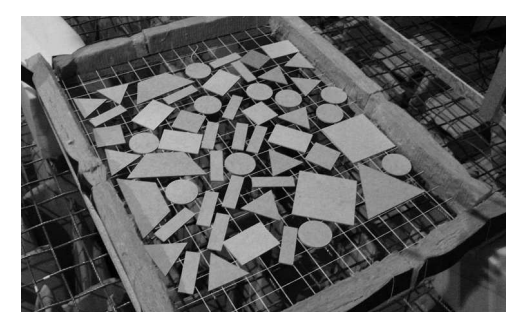

Fig. 8. An array composed of many different elements on the measurement setup.
Figure 7 shows the comparison of two frequency characteristics for square panels. One of them corresponds to regular, and the other to irregular arrangement of elements. The response for the former is extremely rough and unfavorable $\left(L_{\mathrm{pp}}=4.9 \mathrm{~dB}\right)$, while it is fairly even for the latter $\left(L_{\mathrm{pp}}=6.1 \mathrm{~dB}\right)$. Undesirable dips in the first characteristic could be a result of added diffraction effects caused by the periodic pattern. Consequently, irregular arrangement of elements causes mitigation of these phenomena.

The similar result might be obtained for an array composed of many different elements (Fig. 8). The frequency response (Fig. 9) is acceptably smooth in the pass band $\left(L_{\mathrm{pp}}=4.5 \mathrm{~dB}\right)$. Moreover, the cut-off frequency is lower than expected. This may lead to the conclusion that choosing a different panels' shape one may influence the range and the smoothness of frequency response.

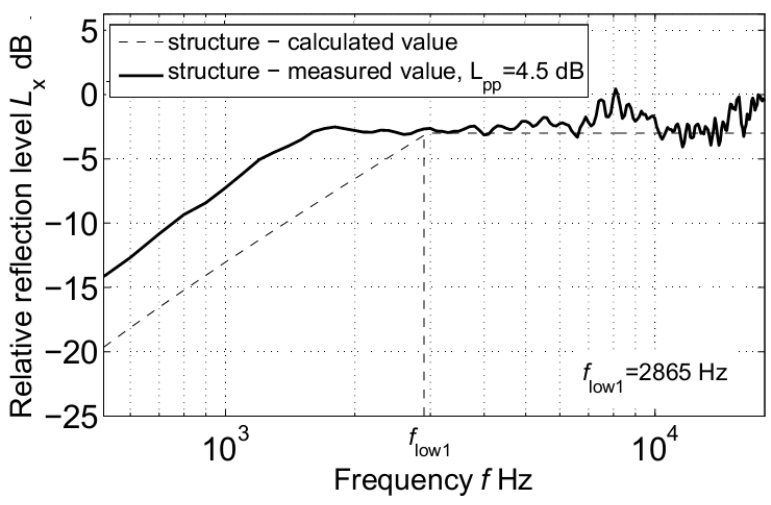

Fig. 9. Frequency responses of reflection arrays composed of many different elements.

\subsection{The influence of element size}

The second important issue in canopy design concerns the size of elements forming a reflection array. In order to lower the cut-off frequency for flat panel arrays, larger elements should be used. Unfortunately, it could cause undesirable strong directional reflection and interference phenomena such as the comb filter.

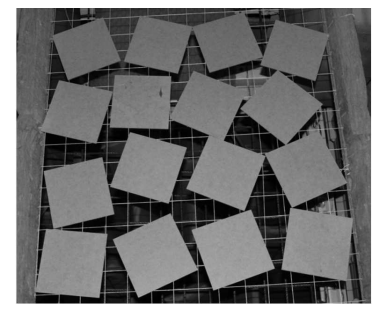

Fig. 10. An array composed of large square elements on the measurement setup.

However, to find the effect of reflection elements' size on frequency response, arrays composed of small (Fig. 6) and large (Fig. 10) square elements were measured. The 


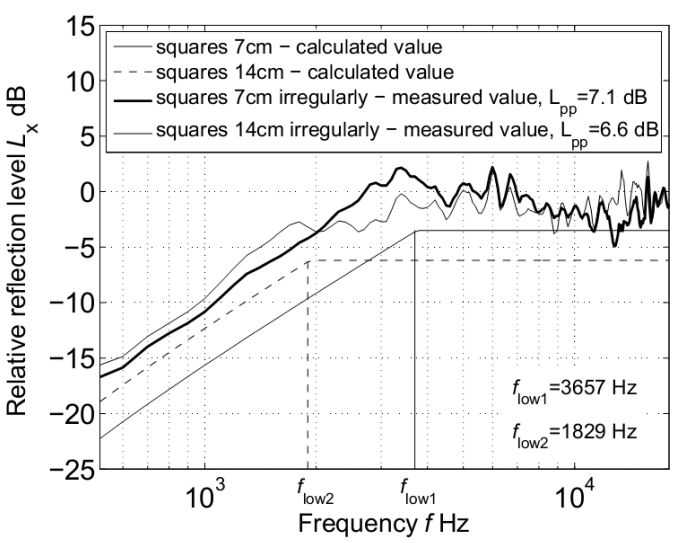

Fig. 11. Frequency responses of reflection arrays composed of small or large square elements.

sides of the square were $7 \mathrm{~cm}$ and $14 \mathrm{~cm}$ long, respectively. The results of the research on these elements are shown in Fig. 11.

Due to the difference between dimensions of both types of panels, the discrepancy between low frequency limits is apparent as well. Besides, according to Rindel's assumption [4], the frequency response for smaller elements should be more even than for bigger ones. However, this is not noticeable enough on the above characteristics, for which the peak-to-peak value is $7.1 \mathrm{~dB}$ and $6.6 \mathrm{~dB}$ respectively.

Another measurement was made for arrays composed of some rectangular elements in various arrangements (Fig. 12).

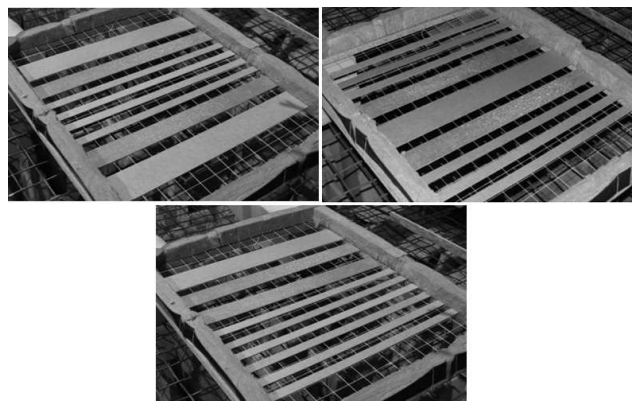

Fig. 12. Arrays composed of rectangular elements in various arrangements on the measurement setup.

All arrays have similar low frequency limits because of their single panels' geometric similarity. Also, the frequency responses (Fig. 13) are similar with the exception of the area around the cut-off frequency. At this point rather strange phenomenon is observed. For the structure with small elements in the middle and large on the verges, the sudden decrease of sound level is noted. In the case of reverse array, the corresponding reflection level is much higher than expected. An intermediate situation occurs for asymmetric structure which can be regarded as a geometric transition between the structures discussed above. In this case, the measured sound level around the cut-off frequency quite accurately coincides with the theoretical value.

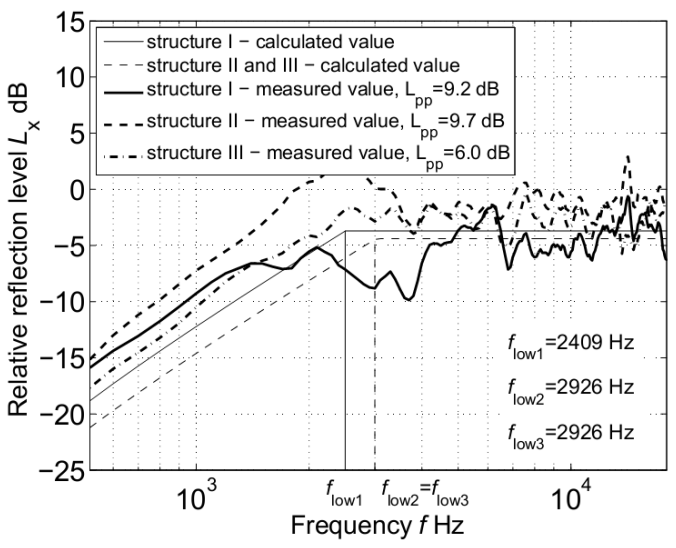

Fig. 13. Frequency responses of reflection arrays composed of rectangular elements in various arrangements.

On the basis of the above measurement one may conclude that by changing the position of elements different in size it is possible to influence the shape of the frequency response.

\subsection{Double-layer array}

For the case when all of the above proposals are insufficient and the use of flat panels only is recommended, Skålevik [7] suggested that a double-layer array could be constructed. It is a two-way system (like in the Oslo Concert Hall), where a small panel array is placed below a larger one (Fig. 14).

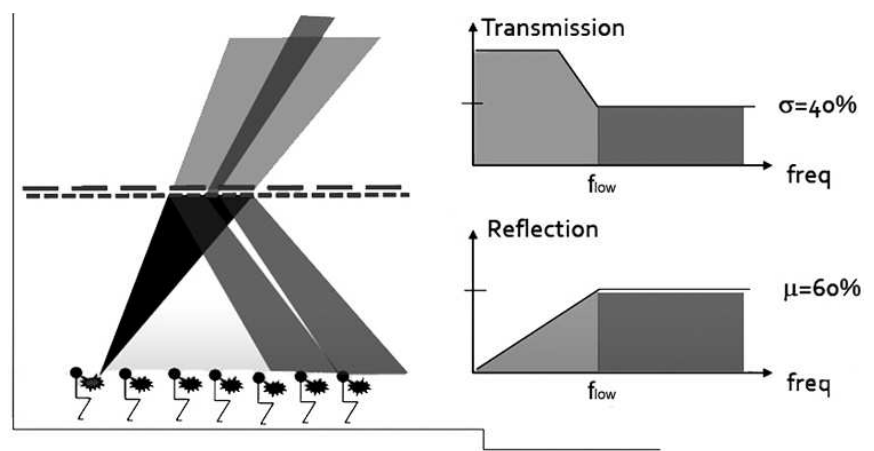

Fig. 14. Sound transmission and reflection for a double-layer array suggested by Skålevik [9].

According to the present authors' paper [8] it is worth noting that few unpredictable problems might occur. In the case of incorrect distance between both arrays, interfering waves may disturb the frequency response in the pass band. A phenomenon known as comb filter may occur which negatively affects the quality of the reflected sound. Results of the research work on single and double structures are shown in Fig. 15. 


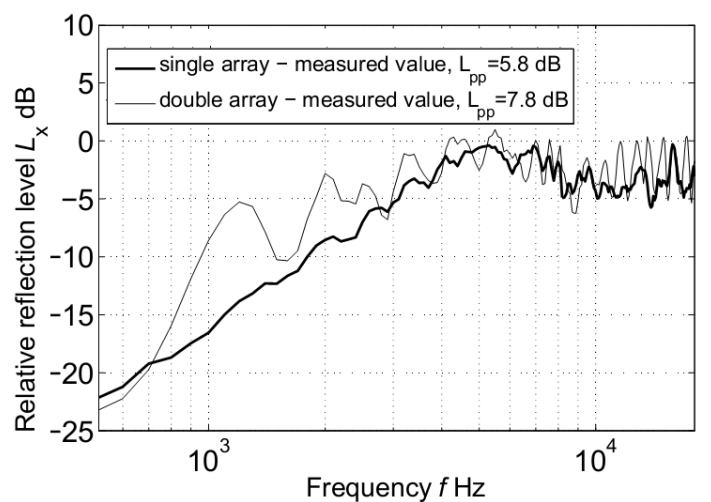

Fig. 15. Frequency responses of single and double reflection arrays.

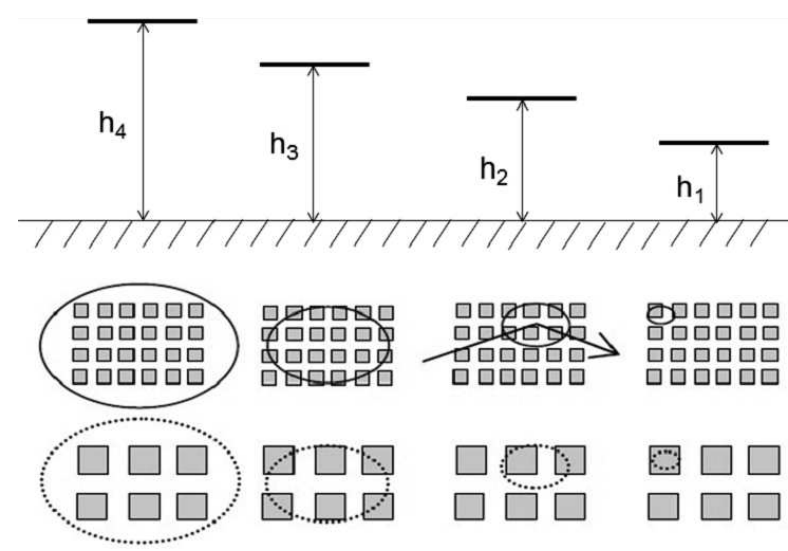

Fig. 16. The Critical Zone dependent on panels' height above the stage.

\subsection{The influence of array height}

Arranging the panels at different heights above the stage could be an alternative solution to narrow sound frequency range problem. With the decrease in distance between a source and a structure, the Critical Zone (similar to the first Fresnel-Zone) also narrows (Fig. 16). Consequently, the reflection from a single panel becomes dominant and the sound level depends on geometric reflection point. Therefore the array composed of panels at different heights provides sound reflection within a wider range of frequency. Lower elements ensure sufficient sound level whilst those above provide the reflection of higher frequencies. Moreover, uneven arrangement of panels favorably influences smoothness of the pass band. The aforementioned theoretical analysis requires experimental verification.

\section{Conclusions and further work}

The paper presents the narrow sound frequency range problem related to flat reflector panels. Mutual depen- dence of the lower and upper frequency limit of sound transmission requires the search for ways to improve these structures. Firstly, the authors suggest that quoted in this paper formulas describing frequency bandwidth should be used with caution and all results require numerical or experimental verification. Moreover, they propose some solutions to spatial structures which have not been yet defined. They show that useful frequency range of sound reflected from these panels closely depends on their shape, size, and configuration. Further, by changing the above parameters one may influence the smoothness of structures' frequency response. Authors also suggest that the element shape has little effect on response smoothness. More important is the spatial arrangement of the elements or their shape variation. Therefore, commonly used identical reflecting panels should be irregularly arranged or replaced with elements differing in shape. Thereby, it is possible to smooth the frequency response even up to $10 \mathrm{~dB}$. Subsequently, lower cut-off frequency for flat panel arrays might be obtained by using larger elements but it causes undesirable strong directional reflection and interference phenomena such as the comb filter. The authors show that by changing the position of elements different in size it is possible to influence the shape of the frequency response. Furthermore, in order to shape the frequency response, double-layer arrays might be used, though they could cause some unfavorable interference phenomena.

All suggestions formulated above are applicable only to flat elements. However, it is sometimes impossible to obtain proper frequency characteristic using planar panels. In such case, other solutions must be taken into account. A natural step further would be to investigate the behavior of curved panels and compare frequency responses of flat, convex and circle elements. One should also study fractal and wavy reflector arrays or panels with diffusers mounted on them [9].

\section{References}

[1] M. Skålevik, IOA Conference on Auditorium Acoustics, Copenhagen 2006.

[2] 2M. Skålevik, ICA 07 Proc., Madrid 2007, p. .

[3] V.O. Knudsen, R.W. Leonard, L.P. Dekasso, J. Acoust. Soc. Amer. 36, 1010 (1964).

[4] J.H. Rindel, Appl. Acoust. 34, 7 (1991).

[5] T. Kamisiński, A. Polaczek, J. Rubacha, Mech. Contr. 29, 174 (2010).

[6] Y. Ando, Architectural Acoustics, Springer Verlag, New York 1998.

[7] M. Skålevik, Joint Baltic-Nordic Acoustics Meeting, Gothenburg 2006, BNAM2006-1.

[8] T. Kamisiński, A. Szeląg, J. Rubacha, Archiv. Acoust. 37, 213 (2012).

[9] T. Kamisiński, K. Brawata, A. Pilch, J. Rubacha, M. Zastawnik, Archiv. Acoust. 37, 317 (2012). 